\title{
Performance and recent developments of the real-time track reconstruction and alignment of the $\mathrm{LHCb}$ detector.
}

\author{
Agnieszka Dziurda*† \\ CERN \\ E-mail: agnieszka.dziurda@cern.ch
}

\begin{abstract}
The $\mathrm{LHCb}$ detector is a single-arm forward spectrometer designed for the efficient reconstruction decays of $c$ - and $b$-hadrons. For Run II (2015-2018) LHCb has introduced a novel real-time detector alignment and calibration strategy. Data collected at the start of the fill are processed in a few minutes and used to update the alignment, while the calibration constants are evaluated for each run. The available timing budget allows the events to be processed using the best performing reconstruction in the trigger, which fully includes particle identification selection criteria. This approach greatly increases the efficiency, in particular for the selection of charm and strange hadron decays.
\end{abstract}

The European Physical Society Conference on High Energy Physics

5-12 July

Venice, Italy

* Speaker.

${ }^{\dagger}$ on behalf of the LHCb collaboration 


\section{The LHCb detector}

The LHCb experiment is dedicated to the study of beauty and charm hadron decays with special attention paid to $\mathrm{CP}$ violating phenomena as well as searches for physics beyond the Standard Model through rare decays. The LHCb detector is a single-arm spectrometer covering the forward pseudorapidty region of $2<\eta<5[1,2]$.

The detector includes a high-precision tracking system consisting of a silicon-strip vertex detector (VELO) surrounding the $p p$ interaction region, a large-area silicon-strip detector (TT) located upstream of a dipole magnet with a bending power of about $4 \mathrm{Tm}$, and three stations of silicon-strip detectors (IT) and straw drift tubes (OT) placed downstream of the magnet commonly known together as $\mathrm{T}$ stations. The particle identification system is composed of two ring-imaging Cherenkov detectors (RICH1, RICH2), electromagnetic (ECAL) and hadronic (HCAL) calorimeters, and a system of muon chambers (MUON). A schematic view of the LHCb detector is shown in Fig. 1 indicating the subdetectors which require to be either aligned or calibrated.

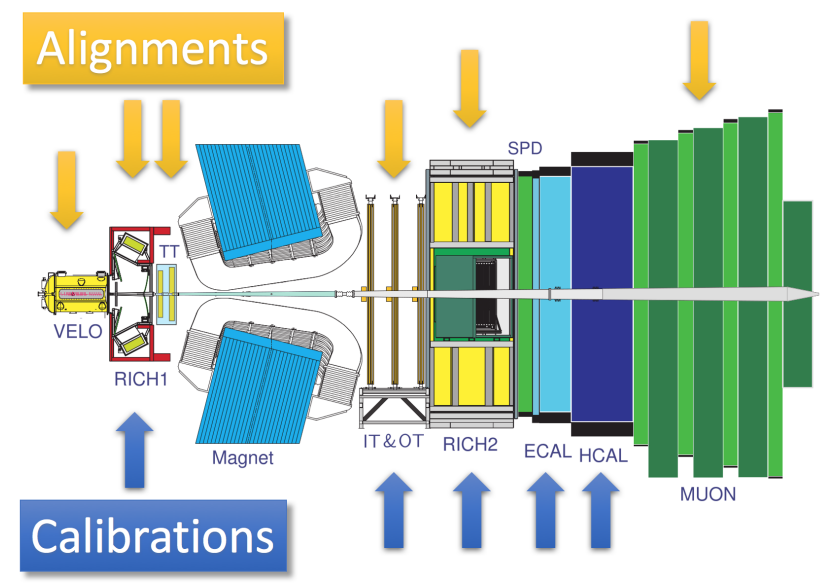

Figure 1: A schematic view of the LHCb detector as described in the text. A blue (orange) arrows indicate the part of the detector which requires calibration (alignment).

\section{Data taking strategy}

In Run II (2015-2018) of data taking the LHCb experiment collects data with the bunch crossing rate of $30 \mathrm{MHz}$. The hardware trigger, based on information from the calorimeter system and muon chambers, reduces the rate of accepted candidates to about $1 \mathrm{MHz}$, at which the full detector can be read out. The candidates accepted by this stage are processed by the high-level software trigger, with two split stages (HLT1 and HLT2). The real-time reconstruction needs to be achieved within 35 (650) ms in the HLT1 (HLT2). All events passing the first stage are buffered on the Event Filter Farm (EFF). Once available, a fraction of these events is used for the run-by-run real-time alignment and calibrations of the detector, resulting in a full, offline quality and best performing event reconstruction achieved in HLT2. 


\section{Real-time alignment and calibration}

The detector alignment and calibration vary due to pressure and/or temperature changes, and movement caused by operational conditions such as switching on the magnetic field or mechanical interventions. To ensure the best physics performance, the position and orientation of detector elements in the global reference frame must be known with an accuracy significantly better than the single hit resolution. The precise alignment of the VELO is crucial for an accurate identification of primary and secondary vertices, and thus better impact parameter (IP), primary vertex (PV) and decay-time resolutions. A more precise alignment of the entire tracking system directly implies a better invariant mass resolution. An accurate calibration and alignment of RICH detectors improves the particle identification and allows to increase the sample purity. The information from the muon system is used in the hardware trigger, where the momentum of muons passing through the detector is estimated from information provided by the first two stations. To guarantee high trigger efficiency without a significant charge asymmetry, an alignment precision better than $1 \mathrm{~mm}$ is required.

The alignment of the tracking system uses an iterative procedure [3, 4] of minimizing the residuals of a Kalman filter and takes into account multiple scattering, energy loss in the material and magnetic field information. The usage of primary vertex and mass constraints minimizes global distortions. The alignment and calibrations are performed at regular intervals, which can be at the beginning of the run, fill or less frequently. The resulting constants are updated only if they differ significantly from the currently used values.

Each automatic alignment procedure runs at the start of each fill when the required sample of candidates has been selected, saved in the buffer and reconstructed within the EFF. Since other subdetectors are aligned relative to the VELO, its alignment is performed first. Figure 2 shows stability of the tracking system alignment for the $\mathrm{x}$ and $\mathrm{y}$ (VELO), $\mathrm{z}$ (Tracker) and $\mathrm{x}$ (MUON) translations during the beginning of 2017 data taking, the horizontal dashed lines indicate the threshold within which the alignment is not updated. Each point denotes the difference between new constants and parameters from the previous alignment. As expected, the constants are updated every few fills and and an overall good stability is found.

\section{Real-time reconstruction}

The LHCb spectrometer has three main tracking subdetectors: VELO, TT and T stations, based on the information from them several type of tracks can be reconstructed: VELO tracks with hits in the VELO; upstream tracks with hits in the VELO and TT; T tracks with hits in the $\mathrm{T}$ stations; downstream tracks with hits in the TT and T stations, which most likely correspond to daughters of long-lived particles. Finally long tracks with hits in at least the VELO and the T stations. Since long tracks pass through the magnetic field, they have the most accurately measured momentum. Due to their properties downstream and long tracks are the most useful for physics analyses. The types of tracks are shown in Fig. 3.

The track reconstruction consists of three main parts. Firstly, the signatures produced in the detector by charged particles are found and the tracks are formed from hits. Then, all found tracks are fitted using a Kalman filter which obtains the best possible estimate of the true trajectory. This 

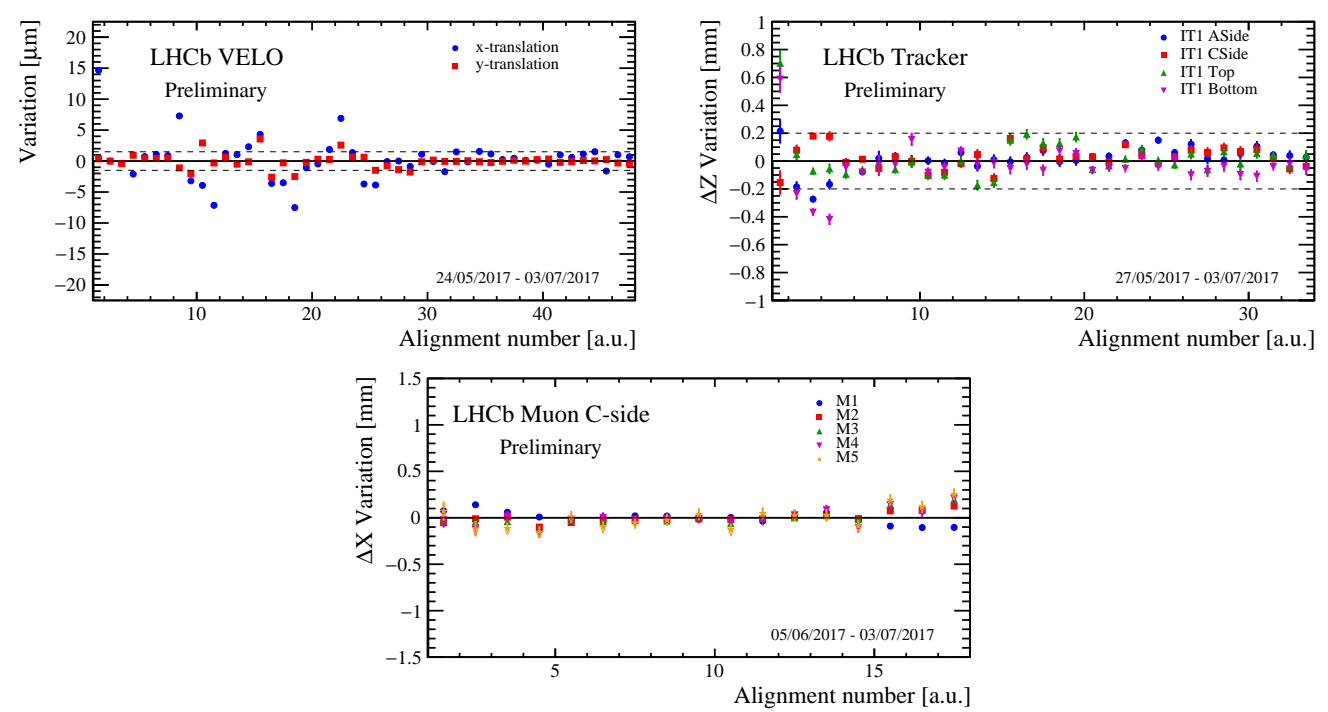

Figure 2: The stability of the VELO half (top left), Tracker (top right) and Muon (bottom) alignment during the beginning of 2017 data taking. Each point indicates the difference between the alignment constants with respect to the value from the previous alignment.

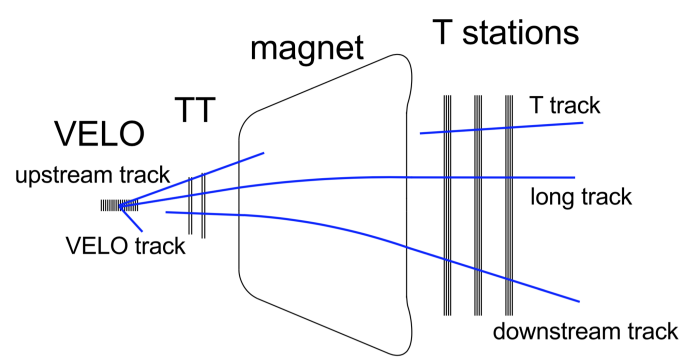

Figure 3: Different type of tracks in the LHCb experiment.

procedure includes corrections due to energy loss and multiple scattering. Finally, any duplicated tracks are removed and a container with the best unique tracks is created.

A strict time budget allowed for the reconstruction in HLT1 and HLT2 stages requires the usage of different track reconstructions. In HLT1, all VELO tracks are reconstructed and fitted with a simplified Kalman filter. These tracks serve as an input to the primary vertex finding, some of them are extended to the TT stations creating upstream tracks with an initial momentum estimation. The upstream tracks are extended further to long tracks by looking for hits in the T stations. The resulting long tracks are Kalman filtered and selected with a transverse momentum threshold above $500 \mathrm{MeV} / \mathrm{c}$. They serve as the necessary input to most of the calibration and alignment algorithms. The second stage, HLT2, runs with looser requirements. Remaining VELO tracks which were not extended into long tracks are propagated to the $\mathrm{T}$ stations, this time without a transverse momentum threshold as well as without requiring clusters in the TT. In addition, $T$ tracks are reconstructed and combined with VELO tracks (long tracks) and with clusters in TT (downstream tracks). All track candidates are fitted with a Kalman filter and clones from different algorithms are removed. 
The LHCb experiment intensively uses the machine learning in the track reconstruction. Neural networks are used in the forward tracking (responsible for finding long tracks) [5] as well and in a dedicated fake track rejection algorithm [6]. In 2017 a new tuning for the downstream tracking has been performed where two different multivariate techniques were employed. Firstly, the algorithm filters $T$ tracks using a bonsai Boosted Decision Tree with 11 dimensional discretized space resulting in a rejection of fake seed tracks. Then the remaining $T$ track candidates are matched with TT hits. Finally, the good track candidates are selected based on a neural network decision. Overall signal efficiency improves by about $O(3-5 \%)$, together with $O(3-5 \%)$ improvement in fake track reduction.

\section{Performance}

Excellent detector performance is achieved as shown in Fig 4. A decay-time resolution of about $45 \mathrm{fs}$ is found for a 4-track vertex. The primary vertex resolution for 25 tracks in $\mathrm{z}$ direction is estimated to be $77 \mu \mathrm{m}$, while the impact parameter resolution in the $\mathrm{x}$ direction is $13 \mu \mathrm{m}$ for tracks with a high transverse momentum. An average tracking efficiency of $>96 \%$ is achieved.
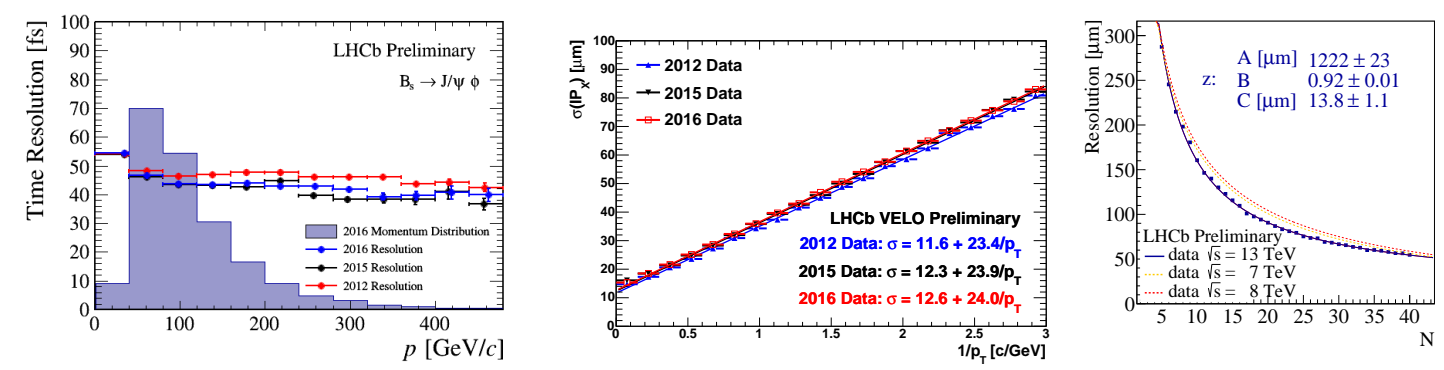

Figure 4: The performance of tracking system. Left: decay-time resolution, middle: impact parameter resolution, right: primary vertex resolution.

\section{Conclusion}

In Run II of data taking the $\mathrm{LHCb}$ detector uses the real-time track reconstruction and alignment. The best quality reconstruction is realised in the trigger resulting in an excellent detector performance.

\section{References}

[1] Alves Jr. et al. (LHCb collaboration), JINST 3, S08005 (2008).

[2] Aaij R. et al. (LHCb collaboration), Int. J. Mod. Phys. A30, 07 1530022, (2015).

[3] Amoraal J. et al., Nucl.Instrum.Meth. A712, 48-55 (2013).

[4] Hulsbergen W.,Nucl.Instrum.Meth., A600, 471-477 (2009).

[5] M.Stahl, LHCb-PROC-2017-013, CERN-LHCb-PROC-2017-013.

[6] P.Seyfert et al, LHCb-PUB-2017-011. 